\title{
Stability of astaxanthin in yogurt used to simulate apricot color, under refrigeration Pedro CEREZAL MEZQUITA ${ }^{1 *}$, Blanca Estela BARRAGÁN-HUERTA², Jennifer PALMA RAMÍREZ ${ }^{1}$, Claudia ORTIZ HINOJOSA ${ }^{1}$
}

\begin{abstract}
The aim of this study was to incorporate astaxanthin to yogurts with different fat content to match apricot (Prunus armeniaca L.) color. The samples containing astaxanthin were stored at $5 \pm 3{ }^{\circ} \mathrm{C}$, and color stability and astaxanthin content were determined by colorimetry and high performance liquid chromatography (HPLC), respectively. Yogurt samples were analyzed in triplicate every 24 hours for one week and subsequently every week for 3 more weeks There were no significant differences $(\mathrm{p}<0.05$ ) between astaxanthin concentration values at 0 and 28 days for both samples; therefore, it can be said that the fat content in the yogurt had not effect on the stability of pigment. The low dispersion of the data showed uniformity in the three chromaticity coordinates $\mathrm{L}^{*}, \mathrm{a}^{*}, \mathrm{~b}^{*}$ throughout the storage period for both types of yogurt. Values of $\Delta \mathrm{E} \geq 5.0$ were not obtained at any time during storage, indicating high stability of the pigment.
\end{abstract}

Keywords: astaxanthin; color; HPLC; yogurt.

\section{Introduction}

At present there is a growing concern among consumers about the consumption of natural foods because people aspire to lead healthier lifestyles. The food industry, aware of this fact, has compiled in a comprehensive manner the effects of food additives on health reported by various agencies, organizations, and national and international institutions regarding artificial pigments and their potential carcinogenic and teratogenic effects and other disorders, especially in children. This has led to the reduction and even the prohibition of artificial pigments in many countries; therefore, the most commonly used synthetic colorants and natural alternatives have been sought as a source of pigments, including components that improve health and welfare and reduce risks of disease.

Pigments are normal constituents of cells or tissues, and they have a physiological function and have some properties that go beyond being colorants, such as energy receptors, oxygen carriers, or protectors against radiation. Among the natural pigments, carotenoids have become increasingly important because they have been used as an alternative to replace synthetic red and yellow colorants, which have been banned by the Food and Drug Administration (FDA) since the 80s (López et al., 2004).

One of the best current sources for the extraction of pigments is microalgae, which have been used over the years for human consumption in various forms such as tablets, capsules, and liquids (Spolaore et al., 2006). They can also be incorporated into pasta, snack, candy, or chewing gum and drinks (Liang et al., 2004). Because of their chemical characteristics, they may act as nutritional supplements or represent a source of natural colorants for food (Borowitzka, 1999). Therefore, microalgae are recognized as an excellent source of natural colorants and nutraceuticals, and it is expected they will surpass synthetics and other natural sources due to their sustainability of production and renewable nature (Dufossé et al., 2005).

Haematococcus pluvialis is a microalgae that synthesizes and accumulates high level of astaxanthin in nature and is one of the natural sources of astaxanthin, which have been widely used in recent years due to its dual activity as antioxidant and colorant (Guerin et al., 2003). Haematococcus sp astaxanthin occurs in three different forms that can be classified as free (5\%), monoesters (70\%), and diesters (25\%), which are difficult to separate, identify, and quantify (Lorenz \& Cysewski, 2000). HPLC analysis of a simple solvent extract of such products would produce many ester-astaxanthin "peaks". This problem is overcome by treating a simple solvent extract of an astaxanthin product with cholesterol esterase, from Pseudomonas flourescens, which gently saponifies the esters (or removes the fatty acids from the astaxanthin), yielding free astaxanthin (Fuji-Chemical Industry Co., 2010; Cysewski, 2006; Cyanotech Corporation, 2001). The enzymatic hydrolysis of carotenoid esters is a slight modification of the procedure developed by Jacobs et al. (1982). Esterified astaxanthin must first be hydrolyzed by either a chemical or enzymatic procedure to yield all free astaxanthin before proceeding with HPLC analysis. However, the enzymatic method is preferable since it is simple, complete, and fast, but the cholesterol esterase is expensive. Several saponification methods under nitrogen for astaxanthin esters have been proposed with the purpose of providing free xanthophylls (Kamata \& Simpson, 1987; Yuan \& Chen, 1999), but standard conditions for the hydrolysis of astaxanthin esters have not been set yet.

Despite the many benefits of astaxanthin as a pigment, there are far few direct applications in food products processed

${ }^{1}$ Departamento de Alimentos, Facultad de Ciencias de la Salud, Universidad de Antofagasta, Avenida Universidad de Antofagasta \# 02800, Campus Coloso, Casilla 170,

Antofagasta, Chile, e-mail: pcerezal@uantof.cl

${ }^{2}$ Departamento de Ingeniería en Sistemas Ambientales, Instituto Politécnico Nacional Delegación Gustavo A. Madero, Ciudad de México, México

${ }^{*}$ Corresponding author 
for human consumption such as oil / water emulsions (Gouveia et al., 2006). It has been widely used in animal feed (Choubert et al., 2009; Ytrestøyl \& Bjerkeng, 2007; Forsberg \& Guttormsen, 2006; Carranco et al., 2003; Lorenz \& Cysewski, 2000). Currently, human consumption of astaxanthin is through the ingestion of nutraceutical capsules, taking into account its antioxidant power (Spolaore et al., 2006). However, researchers are taking the first steps in investigating the addition of astaxanthin in lipidic matrix associated with dairy food, such as flavored milks (Anarjan \& Tan, 2013; Cerezal et al., 2013) and orange juice (Anarjan \& Tan, 2013).

In this study, astaxanthin oleoresin was added to two types of yogurt, "traditional" and "diet" simulating the apricot color. The aim was to determine the content of astaxanthin during the storage of these products by high performance liquid chromatography (HPLC); the chromaticity coordinates were measured using the color space CIEL ${ }^{\star} a^{*} b^{*}$. Based on these measurements, color stability and astaxanthin content were determined in two different lipid matrices.

\section{Materials and methods}

\subsection{Material}

Four different brands (known as A, B, C, D) of the "traditional" apricot flavored yogurt samples (3.4\% total fat) were purchased from the local market. Among them, only the "control samples" were considered those that faithfully reproduce the color of apricot.

Oleoresin samples of natural astaxanthin complex, extracted from the biomass of Haematococcus pluvialis by supercritical $\mathrm{CO}_{2}$ extraction (4.0\% astaxanthin), was kindly donated by the Atacama Bio Natural Products Inc. (2010) Company.

Potassium hydroxide, Sodium chloride, HPLC grade acetone, methanol, water, hexane, and dimethyl sulfoxide (DMSO) were purchased from Merck Co (Chile). Astaxanthin standard 95.5\% was obtained from Dr. Ehrenstorfer $\mathrm{GmbH}$ (Augsburg, Germany).

\subsection{Methods}

Pigmentation of traditional and diet natural yogurt with astaxanthin

A control yogurt sample was selected according to the color preference by a consumer panel using the following parameters: $\mathrm{L}^{\star}$ (a measure of the sample lightness), $\mathrm{a}^{\star}$, a measure of redness $\left(+\mathrm{a}^{*}=\right.$ red, $-\mathrm{a}^{*}=$ green $)$, and $\mathrm{b}^{*}$, a measure of yellowness $\left(+b^{*}=\right.$ yellow, $-b^{*}=$ blue $)$. Subsequently, astaxanthin oleoresin was added in small doses to natural yogurt (without flavor and color) of a selected brand, in the forms traditional and diet with 3.4 and $0.1 \%$ total fat respectively, until reaching $\mathrm{L}^{*}, \mathrm{a}^{*}$ and $\mathrm{b}^{*}$ values similar to those of the control sample. Color determinations were performed using the Hunter Color Instruments (ColorFlex Hunterlab, Virginia, USA), following the operating instructions provided by the manufacturer and the recommended illuminant/observer: $\mathrm{D} 65 / 10^{\circ}$, using the translucent semi-solid sample method.

\section{Storage}

Astaxanthin oleoresin was weighed $(0.055 \pm 0.001 \mathrm{~g})$ and added to $750 \mathrm{~g}$ of natural yogurt, traditional and diet, in a $1000 \mathrm{~mL}$ beaker to match the apricot color of the control sample. It was mixed using an electric mixer (model RW16 Basic, IKA Labortechnik, Brazil) at the speed of $1050 \pm 25 \mathrm{~min}^{-1}$ for $6 \mathrm{~min}$ for the removal of occluded bubbles. When there were no apparent bubbles, it was transferred to a $30 \mathrm{~mL}$ glass container with twist-off caps, previously wrapped in aluminium foil. The samples were stored at $\mathrm{T}=5 \pm 2{ }^{\circ} \mathrm{C}$ in a $90 \mathrm{~L}$ domestic refrigerator of (model FR-093, Daewoo, Argentina).

Colorimetric readings $\left(\mathrm{L}^{*}, \mathrm{a}^{*}\right.$ and $\left.\mathrm{b}^{*}\right)$ and astaxanthin analysis by HPLC were made daily, in triplicate, from day 0 to day 6 , and then on days 14,21 , and 28 for each of the two types of yogurts.

\section{Color analysis}

Color analysis of the two types of yogurt were conducted based on the variations of $L^{*}, a^{*}, b^{*}$, and the color differences $(\Delta \mathrm{E})$ were determined. The determination of $\Delta \mathrm{E}$, was performed using the following Equation (1).

$$
\ddot{A} E=\sqrt{\left(L_{i}^{*}-L_{o}^{*}\right)^{2}+\left(a_{i}^{*}-a_{o}^{*}\right)^{2}+\left(b_{i}^{*}-b_{o}^{*}\right)^{2}}
$$

Where $L_{o}^{*}, a_{0}^{*}$, and $b_{0}^{*}$ are the values of the samples at zero time and $\mathrm{L}_{\mathrm{i}}^{*}, \mathrm{a}_{\mathrm{i}}^{*}$, and $\mathrm{b}_{\mathrm{i}}^{*}$ are the values of each sample with time. $\Delta \mathrm{E}$ comparisons were made based on two different factors: in relation to the color obtained at $t=0$ days with respect to the control sample; and based on its own color at $t=0$ days. The statistical evaluation of the results was performed using common statistical methods. The Excel 2010 spreadsheet software was used in all statistical calculations.

\section{Astaxanthin analysis by HPLC}

HPLC was conducted on the Hitachi L-7100 liquid chromatograph equipped with three pumps and a UV-Vis detector. The pigment extracted and hydrolyzed was analyzed $\left(20 \mu \mathrm{L}\right.$ aliquots) using a $\mathrm{C}_{18}$ column $(250 \times 4.6 \mathrm{~mm} ; 5 \mu \mathrm{m})$ at $25{ }^{\circ} \mathrm{C}$. The mobile phase consisted of mixture of solvent A (acetone), solvent B (methanol), and solvent C (water). For the analysis of free astaxanthin and astaxanthin esters, the following elution procedure was used: 60:23:17 A:B:C v/v for $2 \mathrm{~min}$; linear gradient at 60:30:10 A:B:C v/v for $4 \mathrm{~min}$ and then maintained for 2 more min. The mobile phase was pumped at a flow rate of $1 \mathrm{~mL} / \mathrm{min}$, and the response was detected at $474 \mathrm{~nm}$. The identification of astaxanthin was accomplished by comparison of the retention times with reference standards and co-chromatography with added standards. Freshly prepared Standard Solutions were injected into the HPLC system to construct the standard curve of astaxanthin. Calculation of the astaxanthin concentration was done using the standard curve; which was found to be linear over the required range $\left(1-40 \mu \mathrm{g} / \mathrm{mL} ; \mathrm{R}^{2}=0.9998\right)$. 


\section{Sample preparation for hydrolysis}

Following the procedure reported by Cerezal et al. (2013), known amounts of astaxanthin oleoresin $(0.1 \mathrm{~g})$ were mixed with $2 \mathrm{~mL}$ DMSO and $3 \mathrm{~mL}$ of acetone, $2 \mathrm{~mL}$ brine solution, and $2 \mathrm{~mL}$-hexane in a $15 \mathrm{~mL}$ conical tube. The mixture was shaken using a vortex-mixer (model Classic, VELP Scientifica, Brasil) for 15 seconds and centrifuged (model Mixtasel-BL, JP Selecta S.A., Spain) at $1370 \mathrm{~g}$ for $3 \mathrm{~min}$. Hexane layer containing the carotenoids mixture was removed with a pipette and transferred to a clean tube containing $1.0 \mathrm{~g}$ of anhydrous sodium sulfate. Extractions with $2 \mathrm{~mL}$ of hexane were repeated until the sample was completely clear. The hexane layer was transferred to $50 \mathrm{~mL}$ 24/40 flask, the sodium sulfate crystals were washed with a small amount of hexane until all the carotenoids were recovered and the solvent was eliminated at $40{ }^{\circ} \mathrm{C}$ to dryness using a vacuum rotary evaporator (Model RE-52A, Jiangsu Zhengji Instruments Co., Ltd. China). It was redissolved into $10 \mathrm{~mL}$ of acetone. The acetone extract was adjusted so that the absorbance at $474 \mathrm{~nm}$ ranged between $0.8-1.2$, required for good accuracy in measurement, using a UV/VIS spectrophotometer (model SP-2000, Shanghai Spectrum Instruments Co., Ltd.; China), and $3 \mathrm{~mL}$ aliquots of this extract were used for enzymatic and alkaline hydrolysis. Sample preparation for yogurt requires 4 g portions.

\section{Enzymatic hydrolysis}

The method reported by Cyanotech Corporation (2001) was used with some modifications; in this case, 4 units $/ \mathrm{mL}$ stock of cholesterol esterase enzyme solution in $0.05 \mathrm{M}$ Tris- $\mathrm{HCl}$ $\mathrm{pH} 7.0$ buffer were prepared and maintained at $5 \pm 2{ }^{\circ} \mathrm{C} ; 3 \mathrm{~mL}$ of the solution was transferred to an empty test-tube, and $3 \mathrm{ml}$ of enzyme solution were added to the test tub, which was then capped. They were placed in a water bath accurately regulated to $37^{\circ} \mathrm{C}$ with gentle stirring for $45 \mathrm{~min}$. Thereafter, 1.0 gram of anhydrous sodium sulfate was added, the astaxanthin with $2 \mathrm{~mL}$ of hexane was extracted as mentioned above; the hexane was removed and the sample was redissolved in $3 \mathrm{~mL}$ of acetone and then analyzed by HPLC.

\section{Alkaline hydrolysis}

The method proposed by Yuan \& Chen (1999) was employed with some adjustments. $3 \mathrm{~mL}$ of the extract was transferred to an empty test tube, and the acetone was removed using a vacuum rotary evaporator at $40^{\circ} \mathrm{C}$ and redissolved in $2 \mathrm{~mL}$ of methanol. Next, $0.1 \mathrm{~mL} 1 \% \mathrm{KOH}$ was added and the mixture stirred using a vortex for $1 \mathrm{~min}$. Astaxanthin esters were hydrolyzed at room temperature under $\mathrm{N}_{2}$ and stored in the dark assaying hydrolysis efficiency at $0,0.5,1.0,2.0,4.0$, 6.0 , and 18, 0 hours. The methanol phase was extracted with hexane, and the organic phase was washed with brine solution until $\mathrm{pH}=7$. The hexane extract was dried with anhydrous sodium sulfate, and the solvent was evaporated by flushing with nitrogen gas. The sample was redissolved in $3 \mathrm{~mL}$ of acetone and then analyzed by HPLC.

\section{Statistical analysis}

Experimental results were expressed as mean values \pm standard deviations. The statistical evaluation of the results was performed using common statistical methods and the Microsoft Office Excel 2010 spreadsheet software. Comparison of variance was carried out using one-way analysis of variance (ANOVA), followed up by the Duncan's Multiple Range test for the determination of statistically different groups. Differences were considered significant when $\mathrm{p}<0.05$.

\section{Results and discussion}

\subsection{Pigmentation of natural yogurt, traditional and diet, with astaxanthin}

In order to match the apricot yogurt color, $9.0 \pm 0.1$ mg astaxanthin oleoresin per $125 \mathrm{~g}$ of plain yogurt in two presentations, "traditional" and "diet", was added. With this amount, it was possible to accurately simulate the apricot color of the brand "C", which was chosen by the sensory panel as the one that best represents the apricot color, referred here as "control sample". Chromaticity coordinates values, $\mathrm{L}^{*}, \mathrm{a}^{*}, \mathrm{~b}^{\star}$, of the yogurts pigmented with astaxanthin oleoresin are showed in Table 1. All chromaticity coordinates of the yogurts, traditional and diet, simulating the apricot color in comparison with the apricot control yogurt were significantly different $(\mathrm{p}<0.05)$, except for chromaticity coordinate $\mathrm{a}^{*}$ of the diet yogurt and control yogurt. However, the color differences between the traditional yogurt and the control yogurt, $\Delta \mathrm{E}=2.69$, and between the diet yogurt and the control yogurt, $\Delta \mathrm{E}=1.10$, were lower than $\Delta \mathrm{E}=3.0$, which is a highly satisfactory result since $\Delta \mathrm{E} \leq 3.0$ cannot be detected by the naked human eye (Hong et al., 2004).

Figures $1-4$ show the variations of the chromatic coordinates $\mathrm{L}^{*}, \mathrm{a}^{*}$ and $\mathrm{b}^{*}$ and color differences $(\Delta \mathrm{E})$ obtained

Table 1. Chromaticity coordinates of the apricot yogurt (control sample) and the evaluated yogurts, traditional and diet, pigmented with astaxanthin oleoresin to simulate apricot color $(\mathrm{n}=3)$.

\begin{tabular}{cccc}
\hline & \multicolumn{3}{c}{ Yogurt } \\
\cline { 2 - 4 } Chromaticity coordinates & \multicolumn{2}{c}{ with astaxanthin } & Control \\
\cline { 2 - 4 } & Traditional & Diet & Brand “C” \\
\cline { 2 - 4 } $\mathrm{L}^{*}$ & $83.85 \pm 0.05^{\mathrm{c}}$ & $1.12 \pm 0.14^{\mathrm{a}}$ & $81.40 \pm 0.03^{\mathrm{b}}$ \\
$\mathrm{a}^{*}$ & $10.83 \pm 0.07^{\mathrm{a}}$ & $11.80 \pm 0.17^{\mathrm{b}}$ & $11.81 \pm 0.03^{\mathrm{b}}$ \\
$\mathrm{b}^{*}$ & $16.35 \pm 0.09^{\mathrm{b}}$ & 2.69 & $16.89 \pm 0.01^{\mathrm{c}}$ \\
\hline$\Delta \mathrm{E}$ (Traditional vs Control) & & 1.10 & \\
$\Delta \mathrm{E}$ (Diet vs Control) & & & \\
\hline
\end{tabular}

$\mathrm{X} \pm \mathrm{S}$ : Mean value \pm Standard deviation. Means followed by different superscript letters in each row indicate significant differences at $\mathrm{p} \leq 0.05$. 


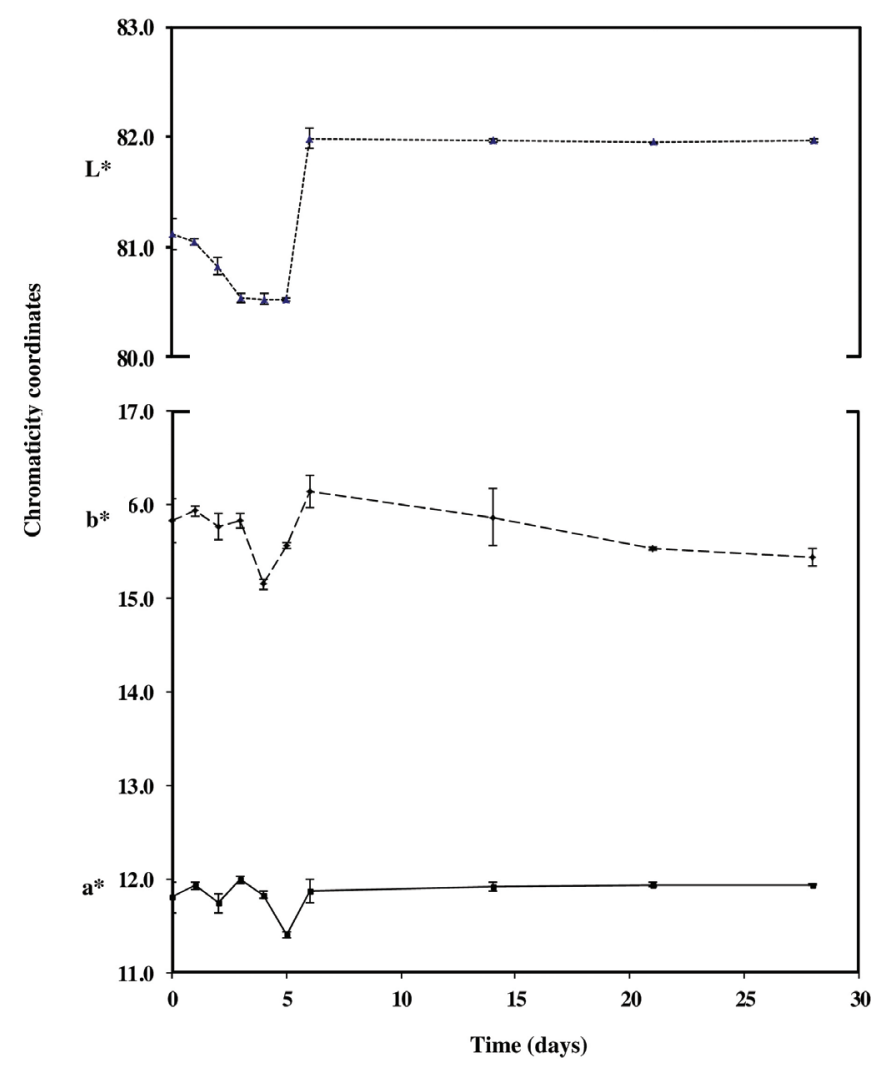

Figure 1. Behavior of Chromaticity coordinates $L^{*}, a^{*}, b^{*}$ of the diet yogurt (apricot color) as a function of storage time using astaxanthin $(n=3)$.

during the storage of 28 days for the traditional and diet yogurts with addition of astaxanthin.

\subsection{Color stability of yogurts during storage}

As can be seen in Figure 1 and Figure 2, the parameter $\mathrm{L}^{*}$ showed a mean value of 81.12 and 83.85 units during the 28 days of storage, indicating that the samples had a high luminosity, similar to the value of $t=0$ days for the control yogurt, $\mathrm{L}^{*}=81.40$ units (Table 1 ). As for the coordinate $\mathrm{a}^{*}$, the mean value of 11.80 and 10.83 units, which is very close to that obtained for the control sample, 11.81 units.

All of these values of the chromatic coordinates $a^{*}$ with positive values were located in the red zone. In the case of the chromaticity coordinate $\mathrm{b}^{*}$, the mean values were 15.83 and 16.35 , slightly lower in relation to the control yogurt (16.89 units), and they are all located on the positive side of the axis, which represents the yellow zone. The samples showed uniformity in the three chromaticity coordinates $\mathrm{L}^{*}, \mathrm{a}^{*}, \mathrm{~b}^{*}$ during the entire storage period for both types of yogurt because of the low dispersion of data.

In Figure 3, $\Delta \mathrm{E}$ value variation was smaller than that in Figure 4 to such an extent that the value of $\Delta \mathrm{E}$ at $\mathrm{t}=0$ days obtained for the sample with added astaxanthin oleoresin and the control sample does not exceed 1.10 units; this result is specifically due to the contribution of the chromatic coordinate

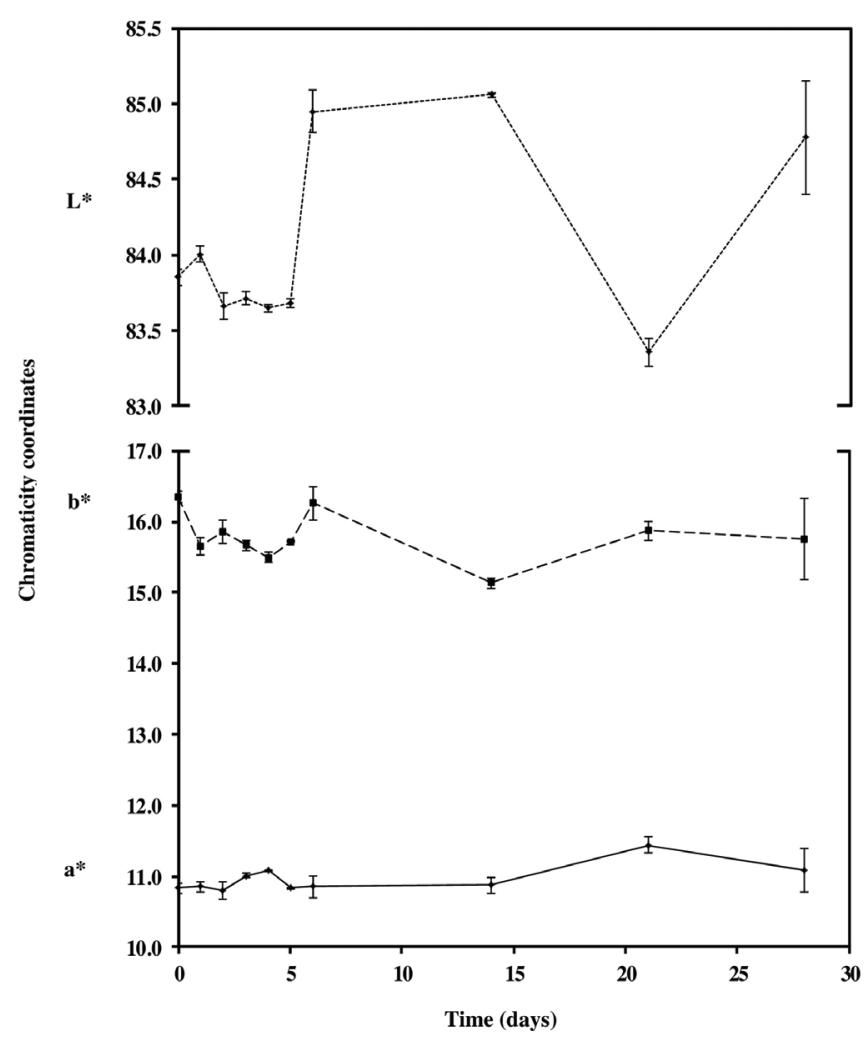

Figure 2. Behavior of Chromaticity coordinates $L^{*}, a^{*}, b^{*}$ of the natural yogurt (apricot color) as a function of storage time using astaxanthin $(n=3)$.

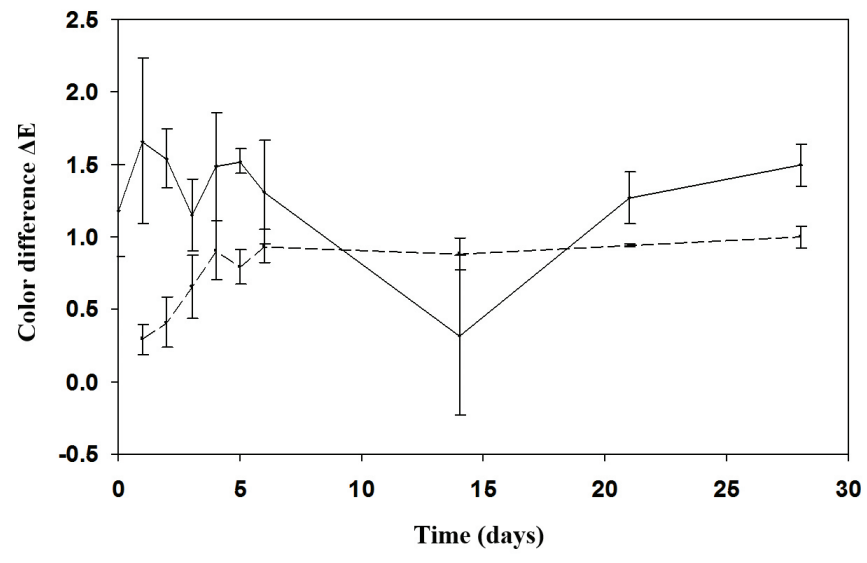

Figure 3. Color difference $(\Delta E)$ of the diet yogurt (apricot color) as a function of storage time using astaxanthin. Comparisons with $t=0$ days of yogurt imitated color (------) and $t=0$ days in the control sample $(-)(\mathrm{n}=3)$.

$\mathrm{b}^{\star}$ between the two samples $\Delta \mathrm{b}^{*}=1.06$ (96.4\%). From that moment until the end of storage period, the effects on the $\Delta \mathrm{E}$ were either produced by $\Delta \mathrm{b}^{*}$ or $\Delta \mathrm{L}^{*} . \Delta \mathrm{E}$ color differences during the entire storage period were $\leq 1.54$, except on day 2 , when $\Delta \mathrm{E}$ was 1.66. However, when comparisons of the sample with astaxanthin were made with the first value obtained at $\mathrm{t}=0$ days, the values of $\Delta \mathrm{E} \leq 1.00$. 


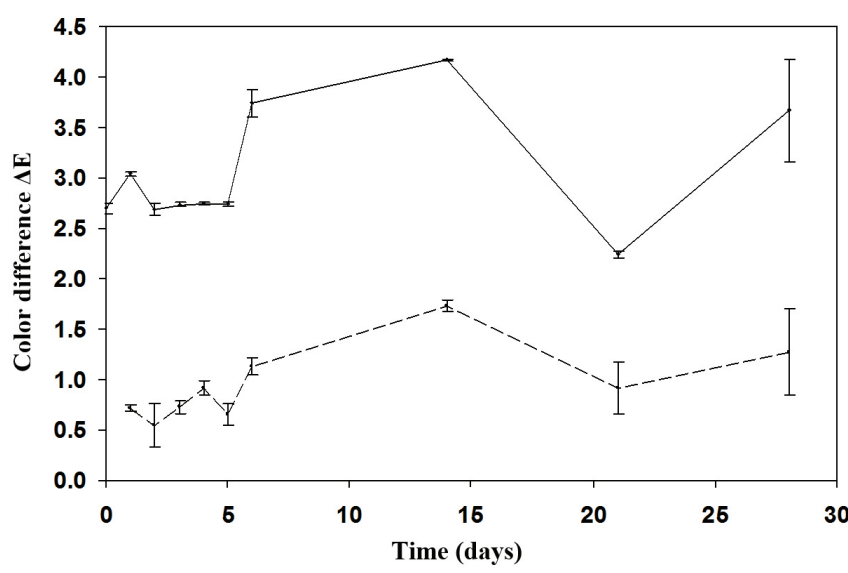

Figure 4. Color difference $(\Delta E)$ of the traditional yogurt (apricot color) as a function of storage time using astaxanthin. Comparisons with $t=0$ days of yogurt imitated color (------) and $t=0$ days in the control sample $(-)(\mathrm{n}=3)$.

In Figure 4 , the $\Delta \mathrm{E}$ value at $\mathrm{t}=0$ days of the sample with astaxanthin and the control sample did not exceed 2.7 units, which is mainly due to the difference established by the contribution of lightness factor $\mathrm{L}^{\star}$ between the two samples $\Delta \mathrm{L}^{*}=2.45$ units $(90.7 \%)$, From then on, it reached values of $\Delta \mathrm{E} \leq 3.70$ during the entire storage period, except on day 14 , when $\Delta \mathrm{E}$ was 4.17 , given by the incidences of clarity $\Delta \mathrm{L}^{*}=3.66$ which contributed to the largest percentage of the color difference $\Delta \mathrm{E}$. However, when comparisons were made between samples with addition of astaxanthin and the control sample in relation to the first value obtained at $\mathrm{t}=0$ days, the values of $\Delta \mathrm{E} \leq 1.73$.

The facts aforementioned indicate that astaxanthin was stable during the entire refrigerated storage period for both the traditional and diet yogurt, without significant changes in any of the three color coordinates. Values of $\Delta \mathrm{E} \geq 5.0$ were not obtained at any time during storage. This a threshold value used by other researchers to indicate the onset of instability (Obón et al., 2009).

The high stability of astaxanthin pigment within the protein-lipid matrix of the yogurts found in this study contrasts with the findings of Krammerer et al. (2006), who pointed out that the colors from natural sources tend to lose tinctorial strength or disappear with time in storage studies.

\subsection{Astaxanthin analysis}

Astaxanthin oleoresin is made up of a complex mixture of colorful components which elute over a broad range of retention (Figure 5a). They correspond mainly to astaxanthin esters of various fatty acids and only approximately $10 \%$ of free astaxanthin. The complexity of the oleoresin makes it difficult to separate all of these components and accurately determinate the content of total astaxanthin and its isomers because only the patterns of pure astaxanthin are commercially available on the market.
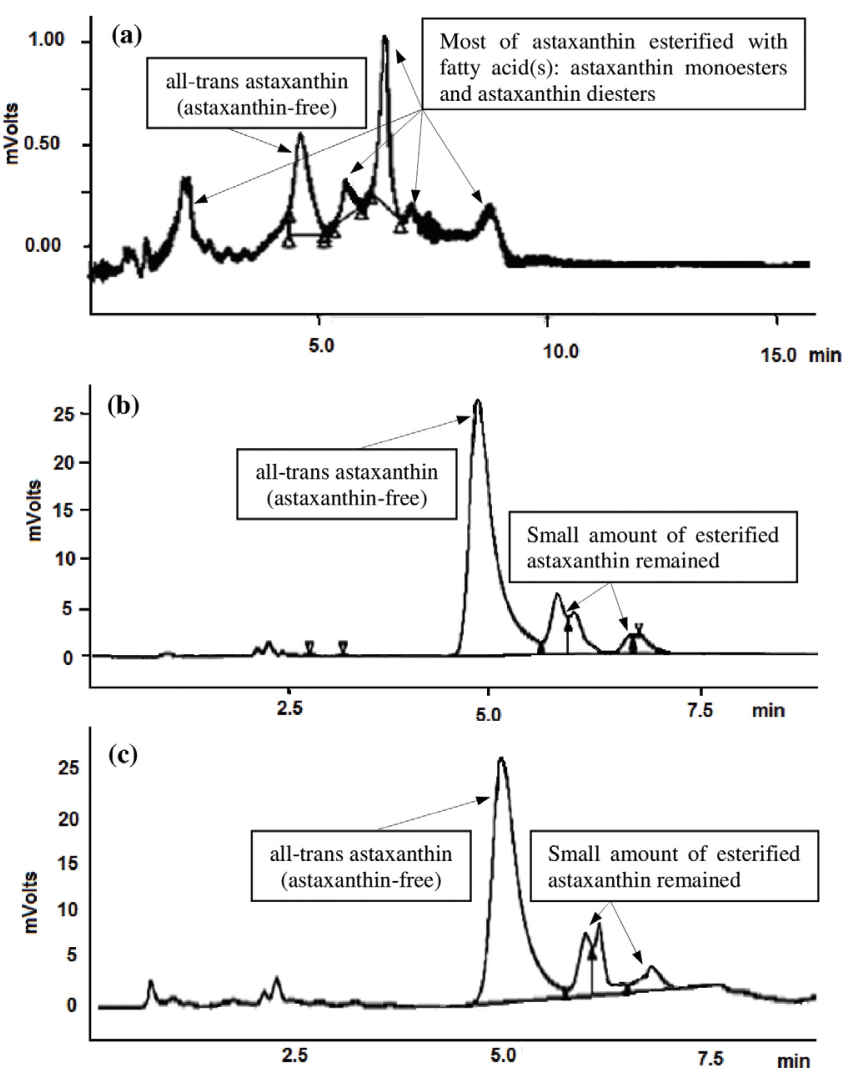

Figure 5. HPLC Chromatograms of astaxanthin oleoresin (a) without hydrolysis, (b) with enzymatic hydrolysis, and (c) with alkaline hydrolysis. Conditions: RP 18 column, $20 \mu \mathrm{L}, 1 \mathrm{~mL} / \mathrm{min}, 474 \mathrm{~nm}$.

This fact explains the need to separate the astaxanthin from its esters to accurately determine its quantification in the oleoresin total Astaxanthin. Enzymatic and alkaline hydrolysis has been suggested to obtain free astaxanthin (Nagao et al., 2003; Yuan \& Chen, 1999). Susceptibility of natural pigments to stress factors, such as temperature, the presence of acids or alkalis, solvents, or light, is well known, and therefore the enzymatic hydrolysis is a recommended method for separating astaxanthin for its quantification (Fuji Chemical Industry Co., 2010).

Therefore, the astaxanthin concentration determinated by cholesterol esterase enzyme in this study is considered maximum. The concentration of free astaxanthin in oleoresin was $3.275 \mathrm{mg}$ of astaxanthin/g oleoresin (Figure 5a). After enzymatic hydrolysis, the monoesters and diesters peaks in the chromatogram are missing (Figure 5b), and astaxanthin concentration increased approximately ten times reaching $38.92 \mathrm{mg}$ of astaxanthin/g oleoresin. Alternatively basic hydrolysis, a cheap and viable alternative method used in this study to free astaxanthin from its esters, resulted in similar enzymatic hydrolysis profile (Figure 5c), with a yield of $37.2 \mathrm{mg}$ astaxanthin/g oleoresin, and enzymatic hydrolysis accounted for $96 \%$.

The results of the kinetic studies of basic hydrolysis to determine the time to obtain the maximum recovery of free astaxanthin from oleoresin are shown in Figure 6. Pigment 


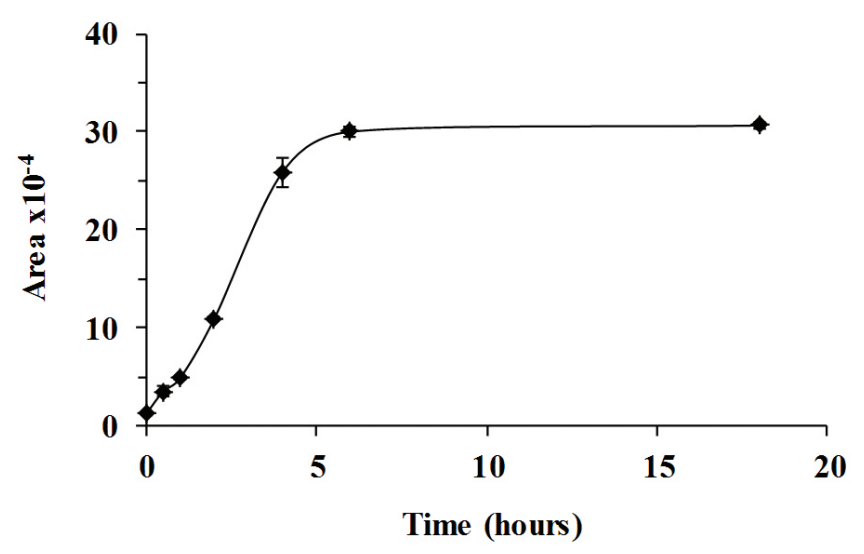

Figure 6. Time course of alkaline hydrolysis with $\mathrm{KOH} 1 \%$ at $22 \pm 2$ ${ }^{\circ} \mathrm{C}(\mathrm{n}=2)$.

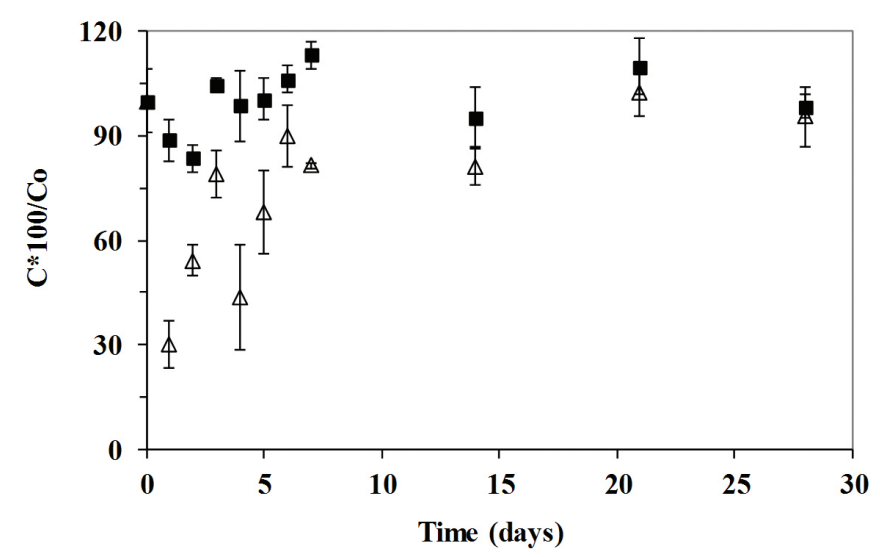

Figure 7. Astaxanthin retention during storage time for the traditional ( $\mathbf{a})$ and $\operatorname{diet}(\Delta)$ yogurts.

concentration increased after $1 \mathrm{~h}$, but the maximum recovery of astaxanthin was observed after 4 hours of hydrolysis, and a stability period started at 6 hours and ended at $18 \mathrm{~h}$. Therefore, the HPLC analyses were conducted considering the total time of $18 \mathrm{~h}$ alkaline hydrolysis.

\subsection{Astaxanthin analysis on storage in pigmented yogurt}

There are many studies in the literature on the use of pigments such as anthocyanin in foods, including dairy products (Giusti \& Wrolstad, 2003), but there is limited information available on carotenoids application, maybe due its low solubility in aqueous matrixes. Application of analytical techniques such as HPLC to ensure the integrity of pigment during storage is not common either, but it is necessary for compounds with biological activity as astaxanthin.

Time course of astaxanthin concentration for both yogurts is shown in Figure 7. There is no significant difference $(\mathrm{p}<0.05)$ between astaxanthin concentration values at 0 and 28 days for both samples, therefore, it can be said that the fat content in the yogurt had not effect on the stability of pigment. These results are in agreement with the colorimetric results, which remained almost constant during storage. In addition, it can be observed that during the first days of storage, samples of traditional yogurt with high concentrations in fat showed dispersion in pigment concentration, which can be due to poor interaction between astaxanthin oleoresin and the lipid phase of the yogurt for the formation of a stable micelle. This dispersion improved during storage and reached a constant value after 21 days. Homogeneity of the diet yogurt sample was maintained during the entire storage period.

\section{Conclusions}

The present study shows that it is possible to use astaxanthin oleoresin to simulate the apricot color in traditional and diet yogurts. The results of the HPLC and colorimetric analysis allowed concluding that this carotenoid pigment is well-packed in the lipid-protein matrix of the final products. In general, the color parameters remained very stable over the four weeks of storage. The results of this study contribute to foster the use of astaxanthin as a functional food justifying the increased production of Haematococcus pluvialis for human consumption.

\section{Acknowledgements}

This study was financially supported by the National Commission for Scientific and Technological Research (CONICYT) of Chile through the Program Featuring Advanced Human Capital from Abroad short-term visits (MEC) (Folio No. 80095006; years 2010 - 2011) and Project Codigue No 2009091 (years 2010 - 2011) belonging to the bilateral program of scientific collaboration between the National Council of Science and Technology (CONACYT) of Mexico and CONICYT of Chile.

\section{References}

Anarjan, N., \& Tan, C. P. (2013). Chemical stability of astaxanthin nanodispersions in orange juice and skimmed milk as model food systems. Food Chemistry, 139(1-4), 527-531. PMid:23561141. http:// dx.doi.org/10.1016/j.foodchem.2013.01.012

Atacama Bio Natural Products Inc. (2010). Iquique, Chile. Retrieved from www.atacamabionatural.com

Borowitzka, M. A. (1999). Commercial production of microalgae: ponds, tanks, tubes and fermenters. Journal of Biotechnology, 70(1-3), 313-321. http://dx.doi.org/10.1016/S0168-1656(99)00083-8

Carranco, M. E., Calvo, C., Arellano, L., Pérez-Gil, F., Ávila, E., \& Fuente, B. (2003). Inclusión de la harina de cabezas de camarón Penaeus sp. en raciones para gallinas ponedoras. Efecto sobre la concentración de pigmento rojo de yema y calidad de huevo. Interciencia, 28(6), 328-333.

Cerezal, P., Barragán, B. E., Palma, J. C., \& Ortíz, C. P. (2013). Milks pigmentation with astaxanthin and determination of colour stability during short period cold storage. Journal of Food Science and Technology. In press.

Choubert, G., Cravedi, J. P., \& Laurentie, M. (2009). Effect of alternate distribution of astaxanthin on rainbow trout (Oncorhynchus mykiss) muscle pigmentation. Aquaculture, 286(1-2), 100-104. http://dx.doi. org/10.1016/j.aquaculture.2008.09.001 
Cyanotech Corporation. (2001). HPLC and spectrophotometric analysis of carotenoids from Haematococcus algae powder (NatuRose Technical Bulletins, Ax-015). Kailua-Kona. 11 p.

Cysewski, G. R. (2006). Analytical methods for measuring Astaxanthin. Kailua-Kona: Cyanotech Corp. Retrieved from www. cyanotech. com/pdfs/axbul20.pdf

Dufossé, L., Galaup, P., Yaron, A., Arad, S. M., Blanc, P., Murthy, K. N. C., \& Ravishankar, G. A. (2005). Microorganisms and microalgae as sources of pigments for food use: a scientific oddity or an industrial reality? Trends in Food Science \& Technology, 16(9), 389-406. http:// dx.doi.org/10.1016/j.tifs.2005.02.006

Forsberg, O. I., \& Guttormsen, A. G. (2006). A pigmentation model for farmed Atlantic salmon: nonlinear regression analysis of published experimental data. Aquaculture, 253(1-4), 415-420. http://dx.doi. org/10.1016/j.aquaculture.2005.09.004

Fuji-Chemical Industry Co., LTD. (2010). Spectrophotometric and HPLC Analysis Method for Determining Astaxanthin Content for AstaREAL(TM)-P2AF. Toyama. Retrieved from www. fujihealthscience.com/Assay\%20Method.pdf

Giusti, M. M., \& Wrolstad, R. (2003). Acylated anthocyanins from edible sources and their applications in food systems. Biochemical Engineering Journal, 14(3), 217-225. http://dx.doi.org/10.1016/ S1369-703X(02)00221-8

Gouveia, L., Raymundo, A., Batista, A. P., Sousa, I., \& Empis, J. (2006). Chlorella vulgaris and Haematococcus pluvialis biomass as colouring and antioxidant in food emulsions. European Food Research and Technology, 222(3-4), 362-367. http://dx.doi.org/10.1007/s00217005-0105-z

Guerin, M., Huntley, M. E., \& Olaizola, M. (2003). Haematococcus astaxanthin: applications for human health and nutrition. Trends Biotechnology, 21(5), 210-216. http://dx.doi.org/10.1016/S01677799(03)00078-7

Hong, S. I., Han, J. H., \& Krochta, J. M. (2004). Optical and surface properties of whey protein isolate coatings on plastic films as influenced by substrate, protein concentration, and plasticizer type. Journal of Applied Polymer Science, 92(1), 335-343. http://dx.doi. org/10.1002/app.20007

Jacobs, P. B., LeBoeuf, R. D., McCommas, S. A., \& Tauber, J. D. (1982). The cleavage of carotenoid esters by cholesterol esterase. Comparative Biochemistry and Physiology Part B: Comparative Biochemistry, 72(1), 157-160. http://dx.doi.org/10.1016/03050491(82)90027-X
Kamata, T., \& Simpson, K. L. (1987). Study of astaxanthin diester extracted from Adonis aestivalis. Comparative Biochemistry and Physiology Part B: Comparative Biochemistry, 86(3), 587-591. http:// dx.doi.org/10.1016/0305-0491(87)90453-6

Krammerer, D., Schillmoller, S., Maier, O., Schieber, A., \& Reinhold, C. (2006). Colour stability of canned strawberries using black carrot and elderberry juice concentrates as natural colorants. European Food Research and Technology, 224(6), 667-669. http://dx.doi. org/10.1007/s00217-006-0356-3

Liang, S., Xueming, L., Chen, F., \& Chen, Z. (2004). Current microalgal health food R\&D activities in China. Hydrobiologia, 512(1), 45-48. http://dx.doi.org/10.1023/B:HYDR.0000020366.65760.98

López, A., García, M., Quintero, R., \& López-Mungía. (2004). Biotecnología alimentaria. México: Limusa. p. 488.

Lorenz, R. T., \& Cysewski, G. R. (2000). Commercial potential for Haematococcus microalgae as a natural source of astaxanthin. Trends Biotechnology, 18(4), 160-167. http://dx.doi.org/10.1016/ S0167-7799(00)01433-5

Nagao, T., Fukami, T., Horita, Y., Komemushi, S., Sugihara, A., \& Shimada, Y. (2003) Enzymatic Enrichment of Astaxanthin from Haematococcus pluvialis Cell Extracts. Journal of the American Oil Chemists Society, 80(10), 975-981. http://dx.doi.org/10.1007/ s11746-003-0806-x

Obón, J. M., Castellar, M. R., Alacid, M., \& Fernández-López, J. A. (2009). Production of a red-purple food colorant from Opuntia stricta fruits by spray drying and its application in food model systems. Journal of Food Engineering, 90(4), 471-479. http://dx.doi. org/10.1016/j.jfoodeng.2008.07.013

Spolaore, P., Joannis-Cassan, C., Duran, E., \& Isambert, A. (2006). Commercial Applications of Microalgae. Journal of Bioscience and Bioengineering, 101(2), 201-211. PMid:16569602. http://dx.doi. org/10.1263/jbb.101.87

Ytrestøyl, T. K., \& Bjerkeng, B. (2007). Dose response in uptake and deposition of intraperitoneally administered astaxanthin in Atlantic salmon (Salmo salar L.) and Atlantic cod (Gadus morhua L.). Aquaculture, 263(1-4), 179-191. http://dx.doi.org/10.1016/j. aquaculture.2006.10.021

Yuan, J. P., \& Chen, F. (1999). Hydrolysis Kinetics of Astaxanthin Esters and Stability of Astaxanthin of Haematococcus pluvialis during saponification. Journal of Agricultural and Food Chemistry, 47(1), 31-35. PMid:10563844. http://dx.doi.org/10.1021/jf980465x 